\title{
Two-Step Magnetic Ordering in Quasi-One-Dimensional Helimagnets: Possible Experimental Validation of Villain's Conjecture about a Chiral Spin Liquid Phase
}

\author{
F. Cinti, ${ }^{1,2}$ A. Rettori, ${ }^{1,2}$ M. G. Pini,${ }^{3}$ M. Mariani, ${ }^{4}$ E. Micotti, ${ }^{4}$ A. Lascialfari,,${ }^{4,5,2}$ N. Papinutto, ${ }^{6}$ A. Amato, ${ }^{7}$ A. Caneschi, ${ }^{8}$ \\ D. Gatteschi, ${ }^{8}$ and M. Affronte ${ }^{2,9}$ \\ ${ }^{1}$ CNISM and Department of Physics, University of Florence, 50019 Sesto Fiorentino (FI), Italy \\ ${ }^{2}$ CNR-INFM S $S^{3}$ National Research Center, Via Campi 213/A, I-41100 Modena, Italy \\ ${ }^{3}$ ISC-CNR, Via Madonna del Piano 10, I-50019 Sesto Fiorentino, Italy \\ ${ }^{4}$ Department of Physics A. Volta and CNR-INFM, University of Pavia, Via Bassi 6, I-27100, Pavia, Italy \\ ${ }^{5}$ Institute of General Physiology and Biological Chemistry, University of Milano, Via Trentacoste 2, I-20134, Milano, Italy \\ ${ }^{6}$ Bruker Biospin s.r.l., Via Pascoli 70/3, I-20133, Milano, Italy \\ ${ }^{7}$ Paul Scherrer Institute, CH-5232 Villingen PSI, Switzerland \\ ${ }^{8}$ INSTM R.U. Firenze and Department of Chemistry, University of Florence, Via della Lastruccia 3, I-50019 Sesto Fiorentino, Italy \\ ${ }^{9}$ Department of Physics, University of Modena and Reggio Emilia, Via Campi 213/A, I-41100 Modena, Italy \\ (Received 28 September 2007; revised manuscript received 23 November 2007; published 6 February 2008)
}

\begin{abstract}
Low-temperature specific heat, magnetic susceptibility, and zero-field muon spin resonance $(\mu \mathrm{SR})$ measurements have been performed in the quasi-one-dimensional molecular helimagnetic compound $\operatorname{Gd}(h f a c)_{3}$ NITEt. The specific heat presents two anomalies at $T_{0}=2.19 \pm 0.02 \mathrm{~K}$ and $T_{N}=1.88 \pm 0.02 \mathrm{~K}$, which both disappear upon the application of a weak magnetic field. Conversely, magnetic susceptibility and $\mu \mathrm{SR}$ data show the divergence of two-spin correlation functions only at $T_{N}=1.88 \pm 0.02 \mathrm{~K}$. These results suggest an experimental validation of Villain's conjecture of a two-step magnetic ordering in quasione-dimensional $X Y$ helimagnets; i.e., the paramagnetic phase and the helical spin solid phase are separated by a chiral spin liquid phase, where translational invariance is broken without violation of rotational invariance.
\end{abstract}

DOI: 10.1103/PhysRevLett.100.057203

Conventional $X Y$ helimagnets (like $\mathrm{Ho}, \mathrm{Tb}, \mathrm{Yb}$ ) have an ordered phase, resulting from the competition of exchange interactions between localized spins, where a $Z_{2} \times \mathrm{SO}(2)$ symmetry is spontaneously broken [1]. In fact, one has to consider as the order parameter not only the spin variable $\vec{S}_{\vec{r}}$, with $\mathrm{SO}(2)$ symmetry, but also the spin chirality [2] $\kappa_{12}=\left[\vec{S}_{\vec{r}_{1}} \times \vec{S}_{\vec{r}_{2}}\right]^{z} / \sin (Q a)(\vec{Q}$ is the pitch of the helical ground state; $\vec{S}_{\vec{r}_{1}}$ and $\vec{S}_{\vec{r}_{2}}$ are spins on nearest neighbor planes perpendicular to $\vec{Q}$, and $a$ is the lattice constant along the chain, $z$ axis). For $T=0, \kappa_{12}= \pm 1$ like an Ising variable, describing the clockwise or anticlockwise degeneracy of the helical structure. If chiral and spin order occur simultaneously, the $Z_{2} \times \mathrm{SO}(2)$ symmetry gives a new universality class for three-dimensional (3D) helimagnets [1]. A different scenario is displayed by another class of helimagnets where the helical modulation is due to a small, yet significant, antisymmetric Dzyaloshinsky-Moriya (DM) interaction: magnetoelectric materials [3-7], and itinerant $\mathrm{MnSi}$ [8-12] and FeGe [13] binary compounds under high pressure. In all these materials, some transitional phases with partial ordering were observed [9] or predicted [7,10-12]. In multiferroic materials, a chiral spin pairing was suggested [7], which has an analogy to a cholesteric liquid crystal; in itinerant helical systems (mainly $\mathrm{MnSi}$ ), where a non-Fermi liquid phase was discovered [8], the partial magnetic order was related to multi- $k$ structure observed in neutron diffraction experi-
PACS numbers: 75.10.-b, 75.30.Kz, 75.40.Cx, 75.50.Xx

ments $[9,14,15]$. Recent theories for these itinerantelectron helimagnets, for which a softening of the amplitude of the magnetization is possible [12], suggest that the partial order is due to the presence of multiple twisted quantum spin structures $[10,11]$ or stable Skyrmion lattices [12], akin to the blue phase of crystal liquids [16].

In a very different context, a model where the chiral symmetry is broken when the system is magnetically disordered was proposed some years ago by Villain [17] for quasi-one-dimensional (1D) $X Y$ helimagnets. Exact transfer matrix calculations for a 1D $X Y$ helimagnet [18] showed that, due to the Ising nature of the chirality order parameter, the chirality-chirality correlation is stronger than the usual spin-spin correlation. The chiral correlation length $\xi_{\kappa},\left\langle\kappa_{1} \kappa_{n+1}\right\rangle \approx \exp \left(-n a / \xi_{\kappa}\right)$, diverges exponentially as temperature is decreased, $\xi_{\kappa} \approx \exp (|J| / T)$ (where $J$ is the exchange constant), while the spin correlation length $\xi_{s},\left\langle\vec{S}_{1} \cdot \vec{S}_{n+1}\right\rangle \approx \exp \left(-n a / \xi_{s}\right)$, diverges as a power law, $\xi_{s} \approx|J| / T$. Taking into account interchain interactions, $J_{\perp}$, within mean-field approximation, Villain [17] predicted a magnetic phase diagram with three phases (see Fig. 1): the usual paramagnetic phase is stable for high temperatures; for $T<T_{0}$ there is a transition to a $3 \mathrm{D}$ chiral spin liquid phase, or spin liquid, given by a collection of corkscrews that turn all clockwise or all anticlockwise, whereas their phases are random; finally, for $T<T_{N}$ a conventional 3D helical spin solid long-range order, or spin solid, is established. As a consequence, an anomaly 


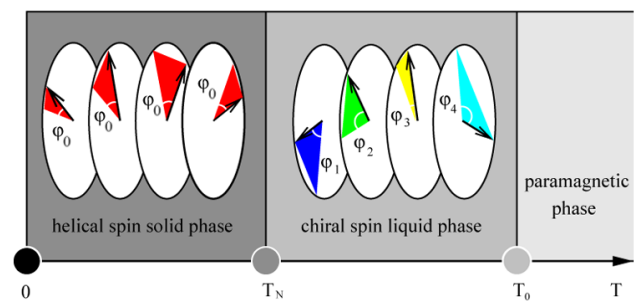

FIG. 1 (color online). Schematic representation of Villain's conjecture. In the chiral spin liquid phase, corkscrews all turning clockwise (or all anticlockwise) with in general $\varphi_{i} \neq \varphi_{j}$. In the helical spin solid phase, same angle value $\varphi_{0}$ for all spins.

at $T_{0}$, due to the onset of chiral long-range order, is expected only for quantities, like the specific heat, related to four-spin correlation functions, while no measurable effect is expected on quantities related to two-spin correlation functions, like the magnetic susceptibility, nuclear magnetic resonance, and muon rotation $(\mu \mathrm{SR})$ spin relaxation. In contrast, at $T_{N}$ an anomaly must be observed in all the above quantities. This prediction seems to be relevant for insulating, molecular-based quasi-1D compounds $\operatorname{Gd}(h f a c)_{3}$ NITR [19,20] [hfac is hexafluoroacetylacetonate, NITR is $2-R-4,4,5,5$-tetramethyl-4,5-dihydro-1H-imidazolyl-1oxil 3 oxide, $R=$ ethyl (Et), isopropyl (iPr), methyl $(\mathrm{Me})$, or phenyl $(\mathrm{Ph})]$. NITR are organic radicals, with spin $|\vec{s}|=1 / 2$, which alternate themselves along the chain $(z)$ direction with $\mathrm{Gd}^{3+}$ magnetic ions, with spin $|\vec{S}|=7 / 2$. The spins interact through competing nearest neighbor ( $\mathrm{NN}), J_{1}>0$, and next nearest neighbor (NNN), $J_{2}<0$ and $J_{2}^{\prime}<0$, exchange couplings, with dominant NNN antiferromagnetic exchange. From EPR spectra, the effective interchain exchange $J_{\perp}$ was estimated to be at least 3 orders of magnitude smaller than the intrachain ones [19]. The Hamiltonian is $\mathcal{H}=$ $\mathcal{H}_{\text {intra }}+\mathcal{H}_{\text {inter }}$, where

$$
\begin{aligned}
\mathcal{H}_{\text {intra }}= & \sum_{i=1}^{M} \sum_{n=1}^{N / 2}\left\{-J_{1}\left(\vec{S}_{i, 2 n-1} \cdot \vec{s}_{i, 2 n}+\vec{s}_{i, 2 n} \cdot \vec{S}_{i, 2 n+1}\right)\right. \\
& -J_{2}\left(\vec{S}_{i, 2 n-1} \cdot \vec{S}_{i, 2 n+1}\right)+D\left(S_{i, 2 n-1}^{z}\right)^{2} \\
& -g \mu_{B} \vec{H} \cdot \vec{S}_{i, 2 n-1}-J_{2}^{\prime}\left(\vec{s}_{i, 2 n} \cdot \vec{s}_{i, 2 n+2}\right) \\
& \left.-g^{\prime} \mu_{B} \vec{H} \cdot \vec{s}_{i, 2 n}\right\}, \\
& \mathcal{H}_{\text {inter }}=\sum_{\langle i, j\rangle} \sum_{n=1}^{N}\left\{-J_{\perp}\left(\vec{X}_{i, n} \cdot \vec{X}_{j, n}\right)\right\},
\end{aligned}
$$

and in Eq. (2) $\vec{X}_{j, n}=\vec{S}_{j, n}, \vec{s}_{j, n} . M$ is the total number of different chains and $N$ the number of spins along a chain (assumed as even); $n$ is the spin position along the $i$ th chain; the sum over $\langle i, j\rangle$ is assumed as limited to NN chains in Eq. (2). $D>0$ is the easy-plane anisotropy; $\vec{H}$ is an external magnetic field. The gyromagnetic factors of the spins $S$ and $s$ are experimentally found to be $g=g^{\prime}=$
2 [19]. Hence we can infer that, even if in these systems the DM interaction is present, it should be very small and probably irrelevant. The Gd(III) ion has the orbital component quenched in the ground ${ }^{8} \mathrm{~S}$ state, and only second order perturbations can allow for small orbital components. In the radicals, which have only light atoms, spin-orbit coupling is small and the largest deviation of the $g$ tensor from the free electron value is 0.01 . Since the DM value can be estimated from $(\Delta g / g) J[21,22]$, an upper limit of $0.1 \mathrm{~K}$ is expected in these $\mathrm{Gd}(h f a c)_{3}$ NITR chain compounds. For the chain with iPr, interesting experimental results were obtained: the specific heat presented a $\lambda$ anomaly for $T=2.08 \mathrm{~K}$, characteristic of a magnetic phase transition, while no evidence for the onset of usual 3D long-range order was shown, at this temperature, by static magnetic susceptibility data (taken for $T \geq 0.2 \mathrm{~K}$ ) [23], neither by $\mu \mathrm{SR}$ (taken for $T \geq 0.3 \mathrm{~K}$ ) [24]. This overall behavior was interpreted in terms of the onset, at $T_{0}=2.08 \mathrm{~K}$, of a chiral spin liquid transition [23,24]. However, in order to complete the validation of Villain's conjecture, also a second phase transition to 3D helical spin solid long-range order for $T_{N}<T_{0}$ should be observed. Recently, we reported a $\lambda$ anomaly for $T=$ $0.039 \mathrm{~K}$, which was tentatively interpreted as the phase transition to the helical spin solid phase [25]. However, this is surprising because, using realistic estimates of the interto-intrachain exchange ratio $r=J_{\perp} / J$ (i.e., $r \geq 10^{-5}$ ), the same order of magnitude is expected for both $T_{N}$ and $T_{0}$ [23]. Furthermore, at these low temperatures, it was not possible to confirm the onset of the 3D long-range order by static magnetic susceptibility and $\mu \mathrm{SR}$ measurements.

In this Letter, we report new experimental results for the quasi-1D molecular magnet $\operatorname{Gd}(h f a c)_{3}$ NITEt, which provide strong support for the validation of Villain's conjecture. In fact, on one hand, the specific heat presents two $\lambda$ anomalies for two comparable temperatures, $T=2.19 \pm$ $0.02 \mathrm{~K}$ and $T=1.88 \pm 0.02 \mathrm{~K}$, and both phase transitions are due to the magnetic degrees of freedom; on the other hand, the static susceptibility and $\mu \mathrm{SR}$ measurements clearly show the onset of a phase transition for $T=$ $1.88 \mathrm{~K}( \pm 0.03 \mathrm{~K}$ for $\chi$ and $\pm 0.1 \mathrm{~K}$ for $\mu$ SR data), but no anomaly at $T=2.19 \mathrm{~K}$. As a consequence, for this $R=$ $E t$ chain compound, we identify $T_{0}=2.19 \mathrm{~K}$ with the chiral spin liquid transition temperature and $T_{N}=1.88 \mathrm{~K}$ with the helical spin solid transition temperature, respectively. In this case $T_{0}$ and $T_{N}$ have the same order of magnitude, a feature that is well reproduced by a meanfield approximation [23] using reasonable values for $J_{\perp}$ (see below). Heat capacity measurements were performed by using a commercial Quantum Design PPMS-7T system by means of a relaxation method with two-tau analysis of the relaxation curves. Microcrystalline samples (between 1 and $2 \mathrm{mg})$ were mixed with a small amount $(\approx 0.3 \mathrm{mg})$ of Apiezon $N$ grease and directly glued on the calorimeter. The heat pulse supplied during the measurement induced 
temperature variations smaller than $1 \%$ of the absolute $T$ value: this determines the accuracy of the measurement, in particular, for the determination of the transition temperature. The inset (a) of Fig. 2 shows the temperature dependence of the specific heat $C$ of $\operatorname{Gd}(h f a c)_{3}$ NITEt, plotted as $C /(R T)$ vs $T^{2}$. Between $4 \mathrm{~K}$ and $10 \mathrm{~K}$, data have an approximatively linear $C /(R T)$ vs $T^{2}$ dependence and they fit the curve $C /(R T)=0.297+0.00295 T^{2}$. A similar behavior was previously observed in the similar compound $\operatorname{Gd}(h \mathrm{fac})_{3}$ NITiPr [23], and it can be explained in terms of a lattice, Debye-like, contribution, $C / R \approx T^{x}$ with $x$ ranging from 2.5 to 3 , plus a quasilinear $C / R \approx T$ term related to the presence of $1 \mathrm{D}$ spin waves. The coefficient is consistent with the values $J_{1}=5.05 \mathrm{~K}, J_{2}=-0.98 \mathrm{~K}$, and $J_{2}^{\prime}=-7.67 \mathrm{~K}$ previously estimated [20]. An excess of specific heat is observed approaching $2 \mathrm{~K}$ and, in zero field, two anomalies are visible [Fig. 2(b)]. The magnetic entropy released around the two transitions is much smaller than the value, $R \ln (16)$, pertinent to this material. This is a well known phenomenon in quasi-1D compounds. For example, in the quasi-1D antiferromagnet $\left(\mathrm{CH}_{3}\right)_{4} \mathrm{NMnCl}_{3}$ the magnetic entropy released around the 3D critical temperature $T_{N}$ is only the $1 \%$ of the total [26]. Both anomalies are completely removed by the application of a weak magnetic field (Fig. 2) and this proves their magnetic origin. The inset Fig. 2(b) shows the excess specific heat estimated by subtracting the $C /(R T)=$ $0.297+0.00295 T^{2}$ contribution from the total specific

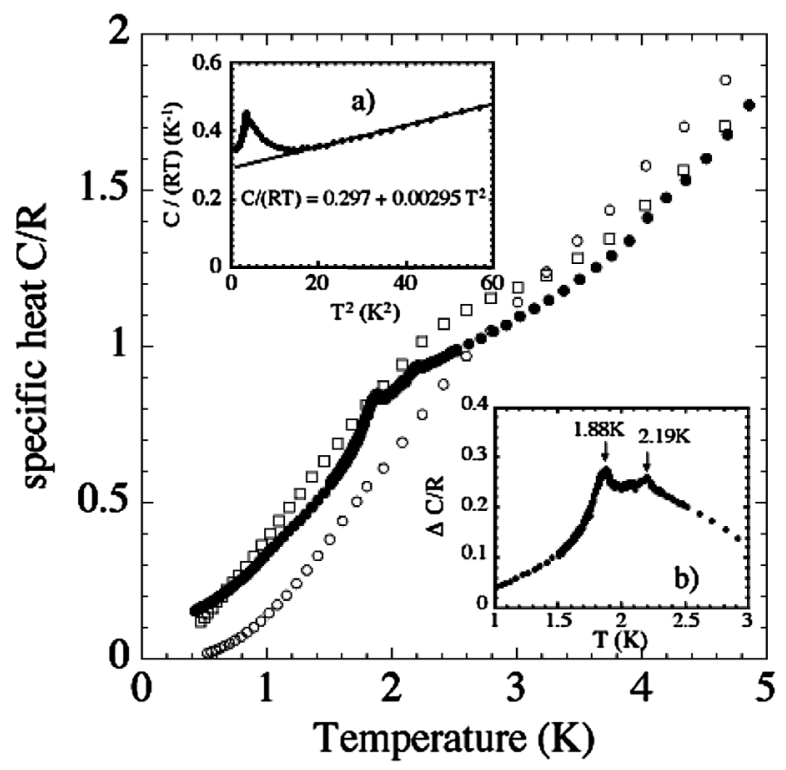

FIG. 2. Temperature dependence of the specific heat of $\operatorname{Gd}(h f a c)_{3}$ NITEt measured in zero field (O) and in external field $H$ of 1000 Oe $(\square)$ and 5000 Oe (O). Inset (a) specific heat $C$, divided by the gas constant $R=8.314 \mathrm{~J} \mathrm{~mol}^{-1} \mathrm{~K}^{-1}$ and by the temperature, of $\operatorname{Gd}(h f a c)_{3}$ NITEt molecular chains plotted as $C /(R T)$ vs $T^{2}$. Inset (b) excess of specific heat, estimated by subtracting the $C / R=0.297 T+0.00295 T^{3}$ contribution from the total $C / R$ value. heat. Two $\lambda$ anomalies are clearly visible at $T=2.19 \pm$ $0.02 \mathrm{~K}$ and $T=1.88 \pm 0.02 \mathrm{~K}$. We have reproducibly found these anomalies in different samples. It is worth noting that the position of the anomalies is also in agreement with the results shown in Ref. [20], even though the origin of these two anomalies was not properly interpreted at that time. We just note that no hysteresis was observed by cycling temperature: this suggests that both phase transitions are of order higher than the first one. In Fig. 3(a) the magnetic susceptibility data are reported as a function of $T$. The measurements were performed using a commercial Quantum Design MPMS-XL7 SQUID magnetometer in the temperature range $1.75 \leq T \leq 300 \mathrm{~K}$. The data were collected by means of the standard dc extraction technique. The magnetic susceptibility $\chi \approx(M / H)_{H \rightarrow 0}(H=5.5 \mathrm{G})$ displays a sharp and sudden change of slope at $T=1.87 \mathrm{~K}$ (see also the inset that shows the anomaly in the quantity $\chi T$ ), while around $T=2.19 \mathrm{~K}$ no anomalous behavior is observed. The low field $H=5.5 \mathrm{G}$ was chosen to have $\chi=M / H$. The field cooled (FC) and zero-field cooled (ZFC) curves coincide, suggesting that in the investigated temperature interval no physical mechanism governed by
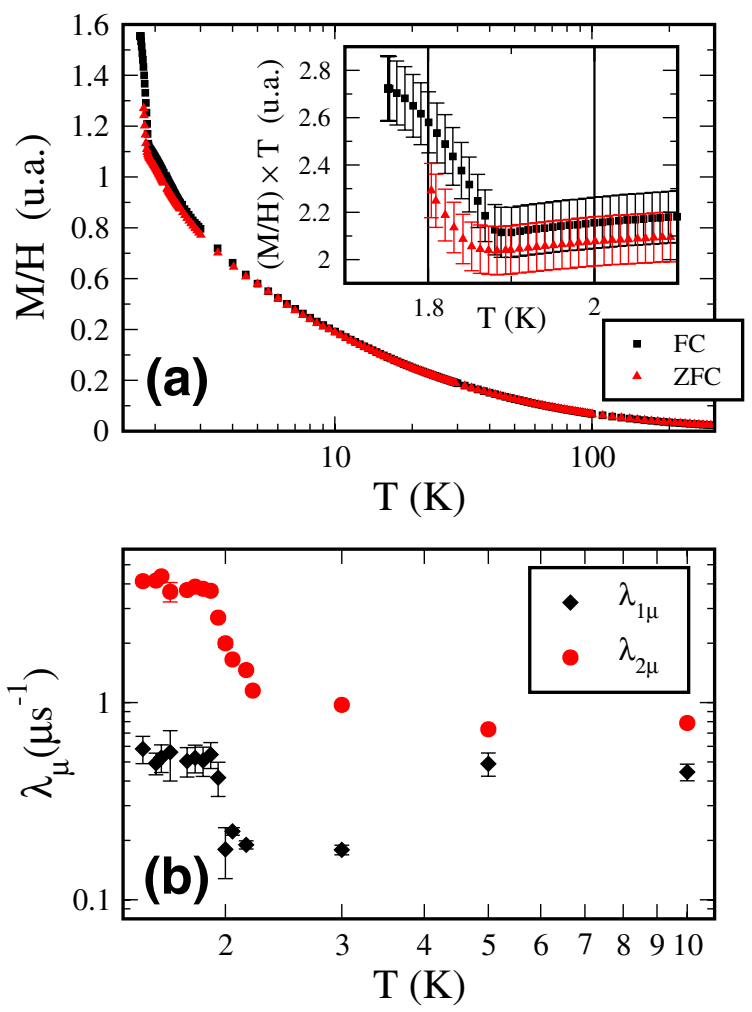

FIG. 3 (color online). (a) Magnetic susceptibility vs temperature of $\mathrm{Gd}(h f a c)_{3}$ NITEt collected in both ZFC and FC modes with external field $H=5.5 \mathrm{Oe}$, from $300 \mathrm{~K}$ to $1.75 \mathrm{~K}$. In the inset the anomaly at $T \sim 1.9 \mathrm{~K}$ in the effective magnetic moment is shown. (b) Fast $\left(\lambda_{1 \mu}\right)$ and slow $\left(\lambda_{2 \mu}\right)$ relaxation rates of a $\operatorname{Gd}(h f a c)_{3}$ NITiEt powder sample, corresponding to two different components of the muon asymmetry, reported as a function of temperature in zero field. 
the slow relaxation of the magnetization [27] is present. Further evidence that quantities related to two-spin correlation functions do not show any anomaly for $T=2.19 \mathrm{~K}$, but only for $T \simeq 1.88 \mathrm{~K}$, is obtained by $\mu \mathrm{SR}$ measurements. They have been performed on a $\operatorname{Gd}(h f a c)_{3}$ NITEt powder sample at the PSI facility in Villigen, on the General Purpose Surface-Muon Instrument beam line. The sample was inserted in a small aluminated Mylar "bag," thus allowing a proper thermalization. The temperature range investigated was $1.5 \leq T \leq 10 \mathrm{~K}$ in zero field. The muon asymmetry, after the usual background subtraction, has been fitted with the sum of two exponential components $a_{0}=a_{1} \exp \left(-\lambda_{1} t\right)+a_{2} \exp \left(-\lambda_{2} t\right)$, where $\lambda_{1}$ and $\lambda_{2}$ represent, respectively, the fast and the slow muon relaxation rates. The occurrence of a biexponential behavior means that the muons implant in (at least) two different interstitial sites. In Fig. 3(b), $\lambda_{1}$ and $\lambda_{2}$ are plotted versus $T$; it is noted that a sudden increase with decreasing temperature occurs below $2.19 \mathrm{~K}$, while a subsequent flattening is evident for $T<1.88 \mathrm{~K}$. This flattening persists till $T=1.5 \mathrm{~K}$, the lowest temperature reached in our measurement. Note that $\lambda_{\text {ratio }}=\lambda_{i \mu}(T=1.88 \mathrm{~K}) / \lambda_{i \mu}(T=$ $3 \mathrm{~K}$ ) is around 4 . Since, in the presence of a phase transition, the relaxation rate presents a divergence of the form $[24,28] \lambda \propto\left(T-T_{N}\right)^{-n}$ where $n=\nu(z-1-\eta)$, with $\nu$, $z$, and $\eta$ the usual critical exponents, from Fig. 3(b) we can conclude that also $\mu \mathrm{SR}$ measurements clearly show a phase transition for $T<1.88 \mathrm{~K}$. The flattening below $T \sim$ $1.88 \mathrm{~K}$ could be due to the presence of low-energy excitations like spin waves, as already detected in this kind of compound [29]. It must be remarked that no precession of the muon polarization below $T=1.88 \mathrm{~K}$ is observed. This experimental evidence led us to conclude that below $T_{N}$ the local spin dynamics is fast in comparison with the typical muon frequencies $\nu_{\mu}$ in a very low applied magnetic field $H$ (zero-field means $H<0.5 \mathrm{mT}$, i.e., $\nu_{\mu}<60 \mathrm{kHz}$ ). From the overall analysis of the experimental data, it appears quite safe to identify $2.19 \mathrm{~K}$ with the temperature $T_{0}$ below which long-range chiral spin liquid order is established, and $1.88 \mathrm{~K}$ with $T_{N}$, below which long-range helimagnetic spin solid order is established. It is worth noting that the experimental critical temperatures can be adequately reproduced, taking into account the interchain interaction within a mean-field approximation [23,30]. Assuming for $J_{1}, J_{2}$, and $J_{2}^{\prime}$ the values above reported and for $J_{\perp} / J_{1}=2.3 \times 10^{-3}$ we find $T_{0}^{\mathrm{MF}}=2.34 \mathrm{~K}$ and $T_{N}^{\mathrm{MF}}=1.90 \mathrm{~K}$.

In conclusion, in this Letter we showed that, in the quasi1D molecular $X Y$ helimagnet $\operatorname{Gd}(h f a c)_{3}$ NITEt, the paramagnetic and helical spin solid phases are separated by a temperature region, $1.88 \mathrm{~K}<T<2.19 \mathrm{~K}$, where a probably ordered chiral spin liquid phase is present. Taking into account the insulating nature of the material, the irrelevance of the DM interaction, and, above all, the quasi-1D nature of the system, we believe we have provided a possible experimental validation of Villain's conjecture about a transitional phase in a $X Y$ helimagnet [17]. It would be very interesting to probe directly the chirality, e.g., using polarized neutron scattering measurements $[31,32]$. Unfortunately, such experiments look unfeasible at the moment since the crystals available so far are too small. Moreover, for the same reasons, it is not possible to obtain accurate estimates of the critical exponents pertinent to the phase transitions investigated here.

This work was supported by EC within the European Network of Excellence MAGMANet under Contract No. 515767.

[1] H. Kawamura, J. Phys. Condens. Matter 10, 4707 (1998).

[2] J. Villain, J. Phys. C 10, 4793 (1977).

[3] M. Mostovoy, Phys. Rev. Lett. 96, 067601 (2006).

[4] H. Katsura and A. V. Bolatsky, Phys. Rev. Lett. 95, 057205 (2005).

[5] I. A. Sergienko and E. Dagotto, Phys. Rev. B 73, 094434 (2006).

[6] T. Arima et al., Phys. Rev. Lett. 96, 097202 (2006).

[7] S. Onoda and N. Nagaosa, Phys. Rev. Lett. 99, 027206 (2007).

[8] C. Pfleiderer et al., Nature (London) 414, 427 (2001).

[9] C. Pfleiderer et al., Nature (London) 427, 227 (2004).

[10] S. Tewari et al., Phys. Rev. Lett. 96, 047207 (2006).

[11] B. Binz et al., Phys. Rev. Lett. 96, 207202 (2006).

[12] U. K. Rößler et al., Nature (London) 442, 797 (2006).

[13] P. Pedrazzini et al., Phys. Rev. Lett. 98, 047204 (2007).

[14] P. Bak and B. Lebech, Phys. Rev. Lett. 40, 800 (1978).

[15] J. R. Stewart et al., J. Phys. Condens. Matter 16, L321 (2004).

[16] D. C. Wright and N. D. Mermin, Rev. Mod. Phys. 61, 385 (1989).

[17] J. Villain, in Proceedings of the 13th IUPAP Conference on Statistical Physics [Ann. Isr. Phys. Soc. 2, 565 (1978)].

[18] I. Harada, J. Phys. Soc. Jpn. 53, 1643 (1984).

[19] C. Benelli et al., Inorg. Chem. 28, 275 (1989); C. Benelli et al., Inorg. Chem. 29, 4223 (1990).

[20] F. Bartolomé et al., Phys. Rev. Lett. 77, 382 (1996); C. Benelli et al., J. Magn. Magn. Mater. 140, 1649 (1995).

[21] T. Moriya, Phys. Rev. 120, 91 (1960).

[22] L. Mihály et al., Phys. Rev. Lett. 97, 067206 (2006).

[23] M. Affronte et al., Phys. Rev. B 59, 6282 (1999).

[24] A. Lascialfari et al., Phys. Rev. B 67, 224408 (2003).

[25] F. Cinti et al., J. Magn. Magn. Mater. 310, 1460 (2007).

[26] M. Steiner et al., Adv. Phys. 25, 87 (1976).

[27] K. Binder and A.P. Young, Rev. Mod. Phys. 58, 801 (1986); J. L. Dormann et al., Adv. Chem. Phys. 98, 283 (1997).

[28] F. Borsa and A. Rigamonti, in Magnetic Resonance at Phase Transitions (Academic Press, New York, 1986).

[29] M. Mariani et al., Inorg. Chim. Acta 360, 3903 (2007).

[30] D. J. Scalapino et al., Phys. Rev. B 11, 2042 (1975).

[31] S. V. Maleyev, Phys. Usp. 45, 569 (2002).

[32] V. P. Plakhty et al., Phys. Rev. B 64, 100402 (2001). 